\title{
A finite-difference scheme for initial boundary value prob- lem of the Gamma equation in the pricing of financial derivatives
}

\author{
Le Minh Hieu a , Truong Thi Hieu Hanh ${ }^{a}$, Dang Ngoc Hoang Thanh ${ }^{b, *}$ \\ a University of Economics, The University of Danang, Vietnam. \\ ${ }^{b}$ Department of Information Systems, School of Business Information Technology, University of Economics Ho Chi Minh city, Vietnam.
}

\begin{abstract}
In the article, we consider the initial boundary value problem for the Gamma equation, which can be derived by transforming the nonlinear Black-Scholes equation for option price into a quasilinear parabolic equation for the second derivative of the option price. We develop unconditionally monotone finite-difference schemes of second-order of local approximation on uniform grids for the initial boundary value problem for the Gamma equation. Two-side estimates of the solution of the scheme are established. By means of regularization principle, the previous results are generalized for construction of unconditionally monotone finite-difference scheme (the maximum principle is satisfied without constraints on relations between the coefficients and grid parameters) of the second-order of approximation on uniform grids for this equation. With the help of difference maximum principle, the two-side estimates for difference solution are obtained at the arbitrary non-sign-constant input data of the problem. A priori estimate in the maximum norm $C$ is proved. It is interesting to note that the proven two-side estimates for difference solution are fully consistent with the differential problem, and the maximal and minimal values of the difference solution do not depend on the diffusion and convection coefficients. Computational experiments, confirming the theoretical conclusions, are given.
\end{abstract}

Keywords: Gamma equation, maximum principle, two-side estimates, monotone finite-difference scheme, quasi-linear parabolic equation, scientific computing.

2010 MSC: 65N06.

(C)2020 All rights reserved.

\section{Introduction}

Over the last decades, not only financial engineers but also mathematicians have paid special attention to the valuation of derivative financial instruments. Indeed, since being introduced by Fischer Black and Myron Scholes in 1973, the Black-Scholes model based on partial differential equation has been widely employed in modern mathematical finance and become a common-sense approach for pricing options as well as many other financial securities [8]. This mathematical model was derived from the principle

\footnotetext{
*Corresponding author

Email addresses: hieulm@due.edu.vn (Le Minh Hieu), hanhtth@due .edu.vn (Truong Thi Hieu Hanh), hoangthanh@ueh.edu.vn, thanh.dnh.cs@gmail.com (Dang Ngoc Hoang Thanh)
}

doi: $10.22436 /$ jmcs.020.04.02

Received: 2019-08-14 Revised: 2020-01-01 Accepted: 2020-01-16 
that yielding profits from making portfolios of both short and long positions in options as well as their underlying stocks should not be possible, if option prices are rightly priced in the market [2]. These scholars indicated that a European options value on a stock, whose price or the log return of underlying price is supposed to follow a geometric Brownian motion with constant volatility and drift, is determined by a second-order parabolic equation concerning time and stock price.

A variety of numerical methods were used in previous papers for studying properties of typical nonlinear BlackScholes equations, see, for instance $[1,3,4]$ and references there in.

Not only in mathematical physics, but also in economics, there is a need to solve partial differential equations containing lower derivatives. For example, in financial mathematics, it is of interest to study the Gamma equation obtained by transforming the nonlinear Black-Scholes equation into a quasilinear parabolic equation [10,11]. The approximate solution of the Gamma equation is the main goal of this study.

In the theory of difference schemes $[9,14,16,18]$, the maximum principle is of great interest. In particular, it is used to study the stability and convergence of a difference solution on a uniform norm. Computational methods that satisfy the maximum principle are called monotone. Monotone schemes play an important role in computational practice, since the corresponding discrete problems are wellposed [7]. Moreover they provide numerical solution without oscillations even in the case of non-smooth solutions [17].

It is non-less important that one can obtain lower estimates of the solutions to differential difference problems, or in the general case, two-sided estimates for the solution of the problems. This is especially important for investigation of theoretical properties of the computational methods approximating problems with unbounded nonlinearities, where it is necessary to prove that discrete solution belongs to a neighborhood of the exact solution. As an example we investigate the Gamma equation modeling pricing of options in financial mathematics. In this context, it is interesting to note the paper [5], in which twosided estimates for solution of difference schemes approximating Dirichlet problem for linear parabolic equation are obtained in the discrete and continuous cases.

In the present paper, the Gamma equation is considered, on the basis of the technique from [12], twosided estimates are obtained for its exact solution. The obtained results are generalized to the construction of unconditionally monotone finite-difference schemes of second-order of local approximation on uniform grids for a given equation. The construction of such schemes is based on the appropriate choice of the perturbed coefficient, similarly to [18]. Using the difference maximum principle, two-sided and a priori estimates are obtained in the C-norm for the difference solution. It is interesting to note that the proved two-sided estimates of the difference solution are completely consistent with the estimates of exact solution of differential problem.

\section{Auxiliary results}

Assume that $\Omega_{h}$ is a finite set of nodes (grid) in some bounded domain of the $n$-dimensional Euclidean space, and $x \in \Omega_{h}$ is a point of the grid $\Omega_{h}$. Consider the equation

$$
A(x) y(x)=\sum_{\xi \in \mathcal{N}^{\prime}(x)} B(x, \xi) y(\xi)+F(x), \quad x \in \Omega_{h \prime}
$$

which is called the canonical form of the finite-difference scheme [18, p. 226]. Here $\mathcal{M}^{\prime}(x)=\mathcal{M}(x) \backslash x$, and $\mathcal{M}(\mathrm{x})$ is the grid stencil. Since any finite-difference scheme can be written as (2.1), monotonicity is understood as the following conditions saying that the coefficients of Eq. (2.1) are positive

$$
\begin{aligned}
& A(x)>0, \quad B(x, \xi)>0 \text { for all } \xi \in \mathcal{M}^{\prime}(x), \\
& D(x)=A(x)-\sum_{\xi \in \mathcal{N}^{\prime}(x)} B(x, \xi)>0 .
\end{aligned}
$$


To obtain a two-sided estimate of the solution of a finite-difference scheme, it is most convenient to use the following lemma.

Lemma 2.1 ([13, 15]). Assume that positivity conditions for coefficients (2.2)-(2.3) are satisfied. Then the maximum and minimum values of the solution of the finite-difference scheme (2.1) belong to the range of the input data

$$
\min _{x \in \Omega_{h}} \frac{F(x)}{D(x)} \leqslant y(x) \leqslant \max _{x \in \Omega_{h}} \frac{F(x)}{D(x)}, \quad x \in \Omega_{h} .
$$

Corollary 2.2 ([18, p. 231]). Assume that conditions of the lemma are satisfied. Then in the grid analog of the C-norm, the solution of finite-difference problem (2.1) satisfies the estimate

$$
\|y\|_{C}=\max _{x \in \Omega_{h}}|y(x)| \leqslant\left\|\frac{F}{D}\right\|_{C} .
$$

\section{Statement of the problem and two-sided estimate of the exact solution}

In a rectangle $\bar{Q}_{\mathrm{T}}=\left\{(x, t): l_{1} \leqslant x \leqslant l_{2}, 0 \leqslant t \leqslant T\right\}$ we consider the following initial boundary value problem for a quasilinear parabolic equation, which is called the Gamma equation [11]

$$
\frac{\partial u}{\partial t}=\frac{\partial^{2} \beta(u)}{\partial x^{2}}+\frac{\partial \beta(u)}{\partial x}+c \frac{\partial u}{\partial x}, \quad u=u(x, t), \quad c=\text { const, }
$$

with homogeneous boundary conditions

$$
u\left(l_{1}, t\right)=u\left(l_{2}, t\right)=0, \quad t>0,
$$

and initial conditions

$$
u(x, 0)=u_{0}(x), l_{1} \leqslant x \leqslant l_{2}
$$

Equation (3.1) can be written as

$$
\frac{\partial u}{\partial t}=\frac{\partial}{\partial x}\left(k(u) \frac{\partial u}{\partial x}\right)+r(u) \frac{\partial u}{\partial x}
$$

with coefficients

$$
k(u)=\beta^{\prime}(u), \quad r(u)=k(u)+c .
$$

We assume that parabolicity condition of equation (3.4) on the solution [6] is satisfied

$$
0<\mathrm{k}_{1} \leqslant \mathrm{k}(\mathrm{u}) \leqslant \mathrm{k}_{2}, \quad \forall \mathrm{u} \in \overline{\mathrm{D}}_{\mathrm{u}}, \quad \mathrm{k}_{1}, \mathrm{k}_{2}=\text { const },
$$

where

$$
\bar{D}_{u}=\left\{u(x, t): m_{1} \leqslant u(x, t) \leqslant m_{2}, \quad(x, t) \in \bar{Q}_{T}\right\} .
$$

We assume in what follows that there exists a unique solution of problem (3.1)-(3.3) and all coefficients in Eq. (3.4) and the desired function have continuous bounded derivatives of order that is required as the presentation proceeds. (3.3).

Using the technique from [12], we prove two-sided estimates for the exact solution of problem (3.1)-

Theorem 3.1. Let condition (3.6) be satisfied. Then for solution $\mathfrak{u}(x, t)$ of problem (3.1)-(3.3) the following twosided estimates are true:

$$
m_{1}=\min \left\{0, \min _{\mathfrak{l}_{1} \leqslant x \leqslant l_{2}} u_{0}(x)\right\} \leqslant u(x, t) \leqslant \max \left\{0, \max _{l_{1} \leqslant x \leqslant l_{2}} u_{0}(x)\right\}=m_{2} .
$$


Proof. To prove (3.7), we make the transformation of the function $u(x, t)$ to the new function $v(x, t)$ associated with it by the equality

$$
u(x, t)=v(x, t) e^{\lambda t}
$$

where $\lambda$ is an arbitrary number. The function $v(x, t)$ satisfies the equation

$$
\frac{\partial v}{\partial t}+\lambda v-k\left(v e^{\lambda t}\right) \frac{\partial^{2} v}{\partial x^{2}}-\frac{\partial k\left(v e^{\lambda t}\right)}{\partial x} \frac{\partial v}{\partial x}-r\left(v e^{\lambda t}\right) \frac{\partial v}{\partial x}=0
$$

with initial and boundary conditions

$$
\begin{aligned}
& v(x, 0)=u_{0}(x), \quad l_{1} \leqslant x \leqslant l_{2}, \\
& v\left(l_{1}, t\right)=v\left(l_{2}, t\right)=0, \quad t>0 .
\end{aligned}
$$

Let the maximum of the solution $v(x, t)$ of problem (3.8)-(3.10) be reached at some point $\left(x_{0}, t_{0}\right) \in\left(l_{1}, l_{2}\right) \times$ $(0, \mathrm{~T}]$

$$
\max _{(x, t) \in \bar{Q}_{T}} v(x, t)=v\left(x_{0}, t_{0}\right),
$$

moreover, at the point $\left(x_{0}, t_{0}\right)$ equation (3.8) and the following relations are satisfied

$$
\frac{\partial v\left(x_{0}, t_{0}\right)}{\partial t}=\frac{\partial v\left(x_{0}, t_{0}\right)}{\partial x}=0, \quad \frac{\partial^{2} v\left(x_{0}, t_{0}\right)}{\partial x^{2}}=\lim _{\Delta x \rightarrow 0} \frac{v\left(x_{0}-\Delta x, t_{0}\right)-2 v\left(x_{0}, t_{0}\right)+v\left(x_{0}+\Delta x, t_{0}\right)}{\Delta x^{2}} \leqslant 0 .
$$

Therefore from equation (3.8) and condition (3.6) it follows that

$$
v(x, t) \leqslant v\left(x_{0}, t_{0}\right)=\frac{1}{\lambda} k\left(v\left(x_{0}, t_{0}\right) e^{\lambda t_{0}}\right) \frac{\partial^{2} v\left(x_{0}, t_{0}\right)}{\partial x^{2}} \leqslant 0, \quad \lambda>0
$$

If the maximal in $\bar{Q}_{T}$ value $v(x, t)$ is taken at the boundary $\left\{l_{1}, l_{2}\right\} \times(0, T] \cup\left[l_{1}, l_{2}\right] \times\{0\}$, then we get

$$
v(x, t) \leqslant \max _{(x, t) \in \bar{Q}_{T}} v(x, t)=\max \left\{0, \max _{l_{1} \leqslant x \leqslant l_{2}} u_{0}(x)\right\} .
$$

Thus, in all cases (3.11)-(3.12) the following estimate is valid

$$
v(x, t) \leqslant \max \left\{0, \max _{l_{1} \leqslant x \leqslant l_{2}} u_{0}(x)\right\}
$$

from which it follows

$$
u(x, t) \leqslant e^{\lambda T} \max \left\{0, \max _{l_{1} \leqslant x \leqslant l_{2}} u_{0}(x)\right\}, \lambda>0 .
$$

When $\lambda \longrightarrow 0$ we get the right-hand side of inequalities (3.7). The case of the minimum of the solution $u(x, t)$ is proved similarly. The theorem is proved.

\section{Unconditionally monotone finite-difference scheme of second order approximation on uniform grids for the Gamma equation} where

Using the principle of regularization [18] on a regular uniform grid in space and time $\bar{\omega}=\bar{\omega}_{h} \times \bar{\omega}_{\tau}$,

$$
\begin{array}{ll}
\bar{\omega}_{h}=\left\{x_{i}=l_{1}+i h, i=0,1,2, \ldots, N=\overline{0, N}, h N=l_{2}-l_{1}\right\}, & \bar{\omega}_{h}=\omega_{h} \cup\left\{x_{0}=l_{1}, x_{N}=l_{2}\right\}, \\
\bar{\omega}_{\tau}=\left\{t_{n}=n \tau, n=\overline{0, N_{0}}, \tau N_{0}=T\right\}, & \bar{\omega}_{\tau}=\omega_{\tau} \cup\left\{t_{N_{0}}=T\right\},
\end{array}
$$


we approximate equation (3.4) with a difference scheme of the form

$$
\begin{aligned}
\frac{y_{i}^{n+1}-y_{i}^{n}}{\tau}= & \frac{k_{i}^{n}(y)}{h}\left(a_{i+1}^{n}(y) \frac{y_{i+1}^{n+1}-y_{i}^{n+1}}{h}-a_{i}^{n}(y) \frac{y_{i}^{n+1}-y_{i-1}^{n+1}}{h}\right) \\
& +b_{i}^{+}(y) a_{i+1}^{n}(y) \frac{y_{i+1}^{n+1}-y_{i}^{n+1}}{h}+b_{i}^{-}(y) a_{i}^{n}(y) \frac{y_{i}^{n+1}-y_{i-1}^{n+1}}{h}, \\
y_{i}^{0}= & u_{0}\left(x_{i}\right), \quad y_{0}^{n+1}=y_{N}^{n+1}=0,
\end{aligned}
$$

where

$$
\begin{aligned}
k_{i}^{n}(y) & =\left(1+R_{i}^{n}(y)\right)^{-1}, & R_{i}^{n}(y) & =0.5 h\left|r\left(y_{i}^{n}\right)\right| / k\left(y_{i}^{n}\right) \geqslant 0, \\
b_{i}^{+}(y) & =r^{+}\left(y_{i}^{n}\right) / k\left(y_{i}^{n}\right) \geqslant 0, & b_{i}^{-}(y) & =r^{-}\left(y_{i}^{n}\right) / k\left(y_{i}^{n}\right) \leqslant 0, \\
r^{+}\left(y_{i}^{n}\right) & =0.5\left(r\left(y_{i}^{n}\right)+\left|r\left(y_{i}^{n}\right)\right|\right) \geqslant 0, & r^{-}\left(y_{i}^{n}\right) & =0.5\left(r\left(y_{i}^{n}\right)-\left|r\left(y_{i}^{n}\right)\right|\right) \leqslant 0, \\
a_{i+1}^{n}(y) & =0.5\left(k\left(y_{i+1}^{n}\right)+k\left(y_{i}^{n}\right)\right), & a_{i}^{n}(y) & =0.5\left(k\left(y_{i-1}^{n}\right)+k\left(y_{i}^{n}\right)\right) .
\end{aligned}
$$

Approximation error. The approximation error of the difference scheme (4.1) has the form

$$
\psi=-u_{t}+k(u)\left(a(u) \hat{u}_{\bar{x}}\right)_{x}+b^{+}(u) a^{(+1)}(u) \hat{u}_{x}+b^{-}(u) a(u) \hat{u}_{\bar{x}},
$$

where

$$
\begin{aligned}
& u=u^{n}=u\left(t_{n}\right), \\
& \hat{u}=u^{n+1}=u\left(t_{n+1}\right), \\
& u_{x}=\left(u_{i+1}-u_{i}\right) / h, \\
& u_{\bar{x}}=\left(u_{i}-u_{i-1}\right) / h \text {, } \\
& a^{(+1)}(u)=a_{i+1}(u), \\
& a(u)=a_{i}(u) \text {. }
\end{aligned}
$$

Taking into account

$$
\begin{aligned}
b^{+}(u) & =r^{+}(u) / k(u), \quad b^{-}(u)=r^{-}(u) / k(u), \\
r^{+}(u)+r^{-}(u) & =r(u), \quad r^{+}(u)-r^{-}(u)=|r(u)|, \\
u_{t} & =\frac{\partial u}{\partial t}+O(\tau), \quad\left(a(u) \hat{u}_{\bar{x}}\right)_{x}=\frac{\partial}{\partial x}\left(k(u) \frac{\partial u}{\partial x}\right)+O\left(h^{2}+\tau\right), \\
a^{(+1)}(u) \hat{u}_{x} & =k(u) \frac{\partial u}{\partial x}+0.5 h \frac{\partial}{\partial x}\left(k(u) \frac{\partial u}{\partial x}\right)+O\left(h^{2}+\tau\right), \\
a(u) \hat{u}_{\bar{x}} & =k(u) \frac{\partial u}{\partial x}-0.5 h \frac{\partial}{\partial x}\left(k(u) \frac{\partial u}{\partial x}\right)+O\left(h^{2}+\tau\right),
\end{aligned}
$$

we get

$$
b^{+}(u) a^{(+1)}(u) \hat{u}_{x}+b^{-}(u) a(u) \hat{u}_{\bar{x}}=r(u) \frac{\partial u}{\partial x}+R(u) \frac{\partial}{\partial x}\left(k(u) \frac{\partial u}{\partial x}\right)+O\left(h^{2}+\tau\right) .
$$

It follows from (4.2) that

$$
\psi=\frac{(R(u))^{2}}{1+R(u)} \frac{\partial}{\partial x}\left(k(u) \frac{\partial u}{\partial x}\right)+O\left(h^{2}+\tau\right)=O\left(h^{2}+\tau\right)
$$

Therefore the following statement is proved.

Theorem 4.1. The difference scheme (4.1) approximating problem (3.1)-(3.3) has second order of approximation with respect to space and first order with respect to time. 


\section{Monotonicity, two-sided and a priori estimates}

We write the difference scheme (4.1) in the canonical form (2.1)

$$
\begin{aligned}
A_{i}^{n} y_{i-1}^{n+1}-C_{i}^{n} y_{i}^{n+1}+B_{i}^{n} y_{i+1}^{n+1} & =-F_{i}^{n}, \quad i=1,2, \ldots, N-1, \\
y_{0}^{n+1} & =y_{N}^{n+1}=0,
\end{aligned}
$$

with coefficients defined as follows

$$
\begin{array}{ll}
A_{i}^{n}=\frac{\tau}{h^{2}} a_{i}^{n}(y)\left(\kappa_{i}^{n}(y)-h b_{i}^{-}(y)\right), & B_{i}^{n}=\frac{\tau}{h^{2}} a_{i+1}^{n}(y)\left(\kappa_{i}^{n}(y)+h b_{i}^{+}(y)\right), \\
C_{i}^{n}=1+A_{i}^{n}+B_{i}^{n}, \quad F_{i}^{n}=y_{i}^{n}, & D_{i}^{n}=C_{i}^{n}-A_{i}^{n}-B_{i}^{n}=1, \quad i=\overline{1, N-1 .}
\end{array}
$$

The scheme (5.1)-(5.2) is monotone if the positivity conditions of the coefficients (2.2)-(2.3) are satisfied [18], i.e.,

$$
A_{i}^{n}>0, \quad B_{i}^{n}>0, \quad D_{i}^{n}=C_{i}^{n}-A_{i}^{n}-B_{i}^{n}>0 .
$$

We need to prove that $a_{i}^{n}(y)>0$ for all $i, n$. In fact, when $n=0$, it is obvious that

$$
a_{i}^{0}(y)=0.5\left(k\left(u_{0 i}\right)+k\left(u_{0 i-1}\right)\right)>0 .
$$

Assume that, for any arbitrary $n, a_{i}^{n}(y)>0$ is also true. From this assumption we have $A_{i}^{n}>0, B_{i}^{n}>0$, $C_{i}^{n}>0$. According to Lemma 1 on the base of the estimate (2.4) for arbitrary $t=t_{n} \in \omega_{\tau}$ and all $i=0,1, \ldots, N$, we have

$$
\min \left\{0, \min _{1 \leqslant i \leqslant N-1} y_{i}^{n}\right\} \leqslant y_{i}^{n+1} \leqslant \max \left\{0, \max _{1 \leqslant i \leqslant N-1} y_{i}^{n}\right\} .
$$

Using induction on $n$, from (5.3) we obtain the two-sided estimate via the input data without assumption for sign-definiteness of input data

$$
\min \left\{0, \min _{l_{1} \leqslant x \leqslant l_{2}} u_{0}(x)\right\} \leqslant y_{i}^{n+1} \leqslant \max \left\{0, \max _{l_{1} \leqslant x \leqslant l_{2}} u_{0}(x)\right\}, \quad i=0,1, \ldots N .
$$

In view of (5.4) we obtain $y_{i}^{n+1} \in \bar{D}_{u}$, i.e., $a_{i}^{n+1}(y)=0.5\left(k\left(y_{i}^{n+1}\right)+k\left(y_{i-1}^{n+1}\right)\right)>0$. Since all positivity conditions for the coefficients (2.2)-(2.3) are satisfied, then the difference scheme (4.1) is monotone for all $h$ and $\tau$ (i.e., unconditionally monotone). Therefore, the following theorem is proved.

Theorem 5.1. Suppose that the conditions (3.6) are fulfilled. Then the finite-difference scheme (4.1) is unconditionally monotone and for its solution $\mathrm{y} \in \overline{\mathrm{D}}_{\mathfrak{u}}$ the above two-sided estimates (5.4) hold.

On the basis of the maximum principle in a standard way we obtain the a priori estimate in the C-norm

Theorem 5.2. Let the condition (3.6) be fulfilled. Then for the solution of the difference problem (4.1) the following a priori estimate holds

$$
\left\|y^{n}\right\|_{\bar{C}} \leqslant\left\|u_{0}\right\|_{\bar{C}} .
$$

Proof. Since all the coefficients of the scheme satisfy inequalities (2.2)-(2.3), on the base of Corollary 2.2 we have $\left\|y^{n+1}\right\|_{\bar{C}} \leqslant\left\|y^{n}\right\|_{\bar{c}}$. Hence, we obtain the chain of relations

$$
\left\|y^{n+1}\right\|_{\bar{C}} \leqslant\left\|y^{n}\right\|_{\bar{C}} \leqslant\left\|y^{n-1}\right\|_{\bar{C}} \leqslant \cdots \leqslant\left\|u_{0}\right\|_{\bar{C}} .
$$

The theorem is proved.

Remark 5.3. It is interesting to note that the maximal and minimal values of the difference solution do not depend on the diffusion coefficient $k(u)$ and the convection coefficient $r(u)$. 
Remark 5.4. For the case of $c=0$, equation (3.4) can be written as

$$
\frac{\partial u}{\partial t}=e^{-x} \frac{\partial}{\partial x}\left(\bar{k}(x, u) \frac{\partial u}{\partial x}\right), \quad \bar{k}(x, u)=e^{x} k(u), \quad k(u)=\beta^{\prime}(u) .
$$

Then, as construction of monotone difference schemes for it, we do not need to use the regularization principle.

Remark 5.5. The estimates obtained in (5.4) are fully consistent with the estimates of exact solution of differential problem (3.7).

An example of the function $\beta(u)$. For the case of the Frey model $[11] \beta(u)=u /(1-\rho u)^{2}, \rho>0$ from (3.5) we obtain the coefficient $k(u)$ of the form $k(u)=(1+\rho u) /(1 \rho u)^{3}$. Then, by virtue of (3.6), equation (3.1) will be parabolic if $k(u)>0, \forall u \bar{D}_{u}$, i.e., if

$$
-\frac{1}{\rho}<u(x, t)<\frac{1}{\rho}
$$

Obviously, for solution of the difference scheme (4.1), which approximates problem (3.1)-(3.3), conditions (5.5) are fulfilled, because by Theorem 3.1 for all $i=0,1,2, \ldots, N, n=0,1,2, \ldots, N_{0}$ we have

$$
-\frac{1}{\rho}<\min \left\{0, \min _{l_{1} \leqslant x \leqslant l_{2}} u_{0}(x)\right\} \leqslant y_{i}^{n} \leqslant \max \left\{0, \max _{l_{1} \leqslant x \leqslant l_{2}} u_{0}(x)\right\}<\frac{1}{\rho} .
$$

\section{Numerical implementation}

\subsection{Example 1.}

We consider the particular case of Gamma equation with homogeneous boundary conditions

$$
\begin{aligned}
\frac{\partial u}{\partial t} & =\frac{\partial}{\partial x}\left(\frac{1+u}{(1-u)^{3}} \frac{\partial u}{\partial x}\right), \quad \rho=1, \quad 0<x<\pi, \quad 0<t \leqslant 1 \\
u(x, 0) & =\sin x, \quad u(0, t)=u(\pi, t)=0 .
\end{aligned}
$$

As the coefficient $k(u)=(1+u) /(1-u)^{3}$ is not defined at $u=1$, then it is not defined for the initial function $u_{0}(x)=\sin x$ at $x=x^{*}=\pi / 2$. So we build uniform grid with step $h=\pi /(2 N+1)$ in order to $x_{i} \neq x^{*}$. The approximate solution of the problem (6.1) at $t=1$, obtained by the difference scheme (4.1), is shown on Fig. 1.

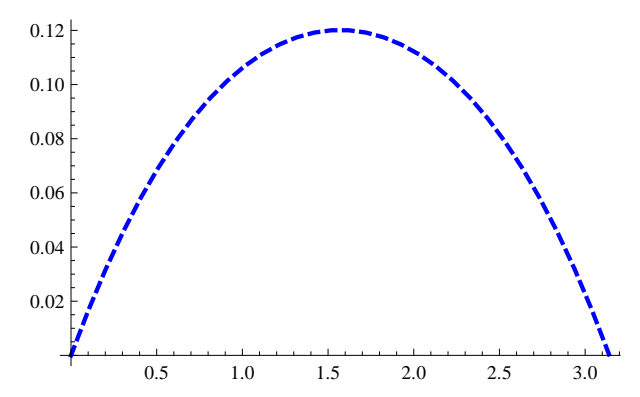

Figure 1: Numerical solution at $t=1$ with step $h=\pi / 31 \approx 0.1$ and $\tau=0.1$.

Remark 6.1. The numerical solution is not defined, if $x=x^{*}$ is a node of the grid. The best numerical results are obtained if the extremal point is not a node of grid. 


\subsection{Example 2.}

Because the Gamma equation has no exact solutions (analytical solutions), to assess efficiency of the proposed difference scheme and to maintain the equality of the Gamma equation, we must add a residual term $f(x, t)$ to the right-hand side of (3.4). We consider equation (3.4) in the form

$$
\frac{\partial u}{\partial t}=\frac{\partial}{\partial x}\left(k(u) \frac{\partial u}{\partial x}\right)+r(u) \frac{\partial u}{\partial x}+f(x, t)
$$

with the boundary and initial conditions

$$
u(-\pi, t)=u(\pi, t)=0, \quad u(x, 0)=u_{0}(x),
$$

and input data

$$
\begin{aligned}
k(u) & =u^{2}+1, r(u)=\sqrt{u+4}, \quad u=u(x, t), \quad x \in[-\pi, \pi], \quad 0 \leqslant t \leqslant T=0.5, \\
f(x, t) & =e^{t}\left(\left(5+e^{2 t}\right) \sin (2 x)-2 \cos (2 x) \sqrt{4+e^{t} \sin (2 x)}-3 e^{2 t} \sin (6 x)\right), \quad u_{0}(x)=\sin (2 x),
\end{aligned}
$$

and suppose that an exact solution is $u(x, t)=e^{t} \sin (2 x)$.

In Table 1 we showed the error of the method in the maximum norm and the $\mathrm{L}^{2}$-norm, respectively,

$$
\begin{aligned}
& \|z\|_{C}=\|y-u\|_{C}=\max _{(x, t) \in \omega}|y(x, t)-u(x, t)|, \\
& \|z\|_{L^{2}}=\|y-u\|_{L^{2}}=\sqrt{\sum_{i=1}^{N-1} h\left(y_{i}-u_{i}\right)^{2}}
\end{aligned}
$$

for different values of space and time discretization parameters. In particular $h=\pi / 10$ with $N=20$ and $\tau=0.1$ have been initially chosen, and from them, $h / 2^{k}$ and $\tau / 4^{k}$, are considered with $k=1,2,3$.

Table 1: Numerical results for problem (6.2)-(6.3).

\begin{tabular}{|l|c|c|c|c|}
\hline & $\mathrm{h}, \tau$ & $\mathrm{h} / 2, \tau / 4$ & $\mathrm{~h} / 4, \tau / 16$ & $\mathrm{~h} / 8, \tau / 64$ \\
\hline$\|z\|_{\mathrm{C}}$ & 0.12364 & 0.0501736 & 0.0132181 & 0.00335072 \\
\hline$\|z\|_{\mathrm{L}^{2}}$ & 0.185965 & 0.0527086 & 0.0136802 & 0.00345762 \\
\hline
\end{tabular}

The approximate solution of the problem (6.2)-(6.3) at $T=0.5$ with step $h=\pi / 20$ and $\tau=0.025$, obtained by the difference scheme (5.1)-(5.2), is shown on Fig. 2.

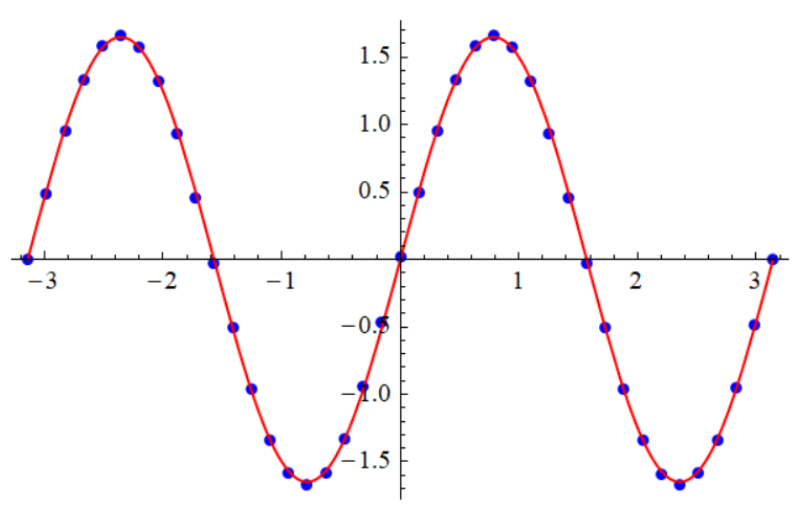

Figure 2: Exact (red line) and approximate (blue nodes) solutions of the problem (6.2)-(6.3) at $\mathrm{T}=0.5$ with step $\mathrm{h}=\pi / 20$ and $\tau=0.025$.

The computational experiment illustrates the higher accuracy of the new scheme and for the scheme (5.1)-(5.2) the accuracy of order $\mathrm{O}\left(\mathrm{h}^{2}+\tau\right)$ is reached on uniform grids in space and time. 


\section{Conclusions}

In the article, we have developed unconditionally monotone finite-difference schemes of second-order of local approximation on uniform grids, based on regularization principle, for the initial boundary problem value for the Gamma equation. Two-side estimates of the solution of the scheme are established. Such estimates permit not only to prove the non-negativity of the exact solution, but also to find sufficient conditions on the input data when the nonlinear problem is parabolic. As a result a priori estimates of the approximate solution in the grid norm $C$ that depend on the initial and boundary conditions only are proved.

\section{Acknowledgements}

This work was supported by University of Economics-The University of Danang (Project T2019-04-43).

\section{References}

[1] G. Barles, H. M. Soner, Option pricing with transaction costs and a nonlinear Black-Scholes equation, Finance Stoch., 2 (1998), 369-397. 1

[2] F. Black, M. Scholes, The pricing of options and corporate liabilities, J. Polit. Econ., 81 (1973), 637-654. 1

[3] R. Company, L. Jódar, E. Ponsoda, C. Ballester, Numerical analysis and simulation of option pricing problems modeling illiquid markets, Comput. Math. Appl., 59 (2010), 2964-2975. 1

[4] E. Dremkova, M. Ehrhardt, A high-order compact method for nonlinear Black-Scholes option pricing equations of American options, Int. J. Comput. Math., 88 (2011), 2782-2797. 1

[5] I. Faragó, R. Horváth, Discrete maximum principle and adequate discretizations of linear parabolic problems, SIAM J. Sci. Comput., 28 (2006), 2313-2336. 1

[6] A. Friedman, Partial Differential Equations of Parabolic Type, Prentice-Hall, Englewood Cliffs, (1964). 3

[7] S. K. Godunov, V. S. Ryaben'kii, Difference Schemes (in Russian), Nauka, Moscow, (1977). 1

[8] O. Hyong-Chol, J. J. Jo, J. S. Kim, General properties of solutions to inhomogeneous Black-Scholes equations with discontinuous maturity payoffs, J. Differential Equations, 260 (2016), 3151-3172. 1

[9] L. M. Hieu, D. N. H. Thanh, S. Prasath, Second order monotone difference schemes with approximation on non-uniform grids for two-dimensional quasilinear parabolic convection-diffusion equations, Vestnik St. Petersburg University, Mathematics, 53 (2020), (In Press). 1

[10] M. Jandačka, D. Ševčovič, On the risk-adjusted pricing-methodology-based valuation of vanilla options and explanation of the volatility smile, J. Appl. Math., 3 (2005), 235-258. 1

[11] M. N. Koleva, L. G. Vulkov, A second-order positivity preserving numerical method for Gamma equation, Appl. Math. Comput., 220 (2013), 722-734. 1, 3, 5

[12] O. A. Ladyžhenskaya, V. A. Solonnikov, N. N. Ural'ceva, Linear and Quasilinear Equations of Parabolic Type (in Russian), Nauka, Moscow, (1967). 1, 3

[13] P. Matus, L. M. Hieu, L. G. Vulkov, Maximum principle for finite-difference schemes with non sign-constant input data (in Russian), Dokl. Nats. Akad. Nauk Belarusi, 59 (2015), 13-17. 2.1

[14] P. Matus, L. M. Hieu, D. Pylak, Monotone finite-difference schemes of second-order accuracy for quasilinear parabolic equations with mixed derivatives, Diff. Equ., 55 (2019), 424-436. 1

[15] P. Matus, L. M. Hieu, L. G. Vulkov, Analysis of second order difference schemes on non-uniform grids for quasilinear parabolic equations, J. Comput. Appl. Math., 310 (2017), 186-199. 2.1

[16] P. Matus, D. Poliakov, L. M. Hieu, On convergence of difference schemes for dirichlet IBVP for two-dimensional quasilinear parabolic equations with mixed derivatives and generalized solutions, Comput. Meth. Appl. Math., (2020) (In Press). 1

[17] P. P. Matus, V. T. K. Tuyen, F. Z. Gaspar, Monotone difference schemes for linear parabolic equations with mixed boundary conditions (in Russian), Dokl. Nats. Akad. Nauk Belarusi, 58 (2014), 18-22. 1

[18] A. Samarskii, The Theory of Difference Schemes, Marcel Dekker, New York, (2001). 1, 2, 2.2, 4, 5 